\title{
Simultaneous Casualty Admissions-Do they Affect Treatment in the Receiving Trauma Center?
}

\author{
Michel Paul Johan Teuben ${ }^{1,2} \cdot{\text { Carsten } \text { Mand }^{3} \cdot \text { Laura Moosdorf }^{3} \cdot \text { Kai Sprengel }}^{1} \cdot$ Alba Shehu $^{1}$ • \\ Roman Pfeifer $^{1} \cdot$ Steffen Ruchholtz $^{3} \cdot$ Rolf Lefering $^{4} \cdot$ Hans-Christoph Pape ${ }^{1} \cdot$ Kai Oliver Jensen $^{1}$
}

\begin{abstract}
Background Simultaneous trauma admissions expose medical professionals to increased workload. The impact of simultaneous trauma admissions on hospital allocation, therapy, and outcome is currently unclear. We hypothesized that multiple admission-scenarios impact the diagnostic pathway and outcome.

Methods The TraumaRegister DGU® was utilized. Patients admitted between 2002-2015 with an ISS $\geq 9$, treated with ATLS $\left({ }^{\circledR}\right.$ - algorithms were included. Group 'IND' included individual admissions, two individuals that were admitted within $60 \mathrm{~min}$ of each other were selected for group 'MULT'. Patients admitted within 10 min were considered as simultaneous ('SIM') admissions. We compared patient and trauma characteristics, treatment, and outcomes between both groups.

Results 132,382 admissions were included, and 4,462/3.4\% MULTiple admissions were found. The SIM-group contained $1,686 / 1.3 \%$ patients. The overall median injury severity score was 17 and a mean age of 48 years was found. MULT patients were more frequently admitted to level-one trauma centers $(68 \%)$ than individual trauma admissions were $(58 \%, \mathrm{p}<0.001)$. Mean time to CT-scanning (24 vs. $26 / 28 \mathrm{~min}$ ) was longer in MULT / SIM patients compared to individual admissions. No differences in utilization of damage control principles were seen. Moreover, mortality rates did not differ between the groups $(13.1 \%$ in regular admissions and $11.4 \% / 10,6 \%$ in MULT/SIM patients).

Conclusion This study demonstrates that simultaneous treatment of injured patients is rare. Individuals treated in parallel with other patients were more often admitted to level-one trauma centers compared with individual patients. Although diagnostics take longer, treatment principles and mortality are equal in individual admissions and simultaneously admitted patients. More studies are required to optimize health care under these conditions.
\end{abstract}

This study was presented at the ESTES-Conference 05/2019 in Prague, Czech Republic and at the American College of Surgeons Clinical Congress 10/2020 and at the ATLS European Association Annual Meeting 09/2020.

Kai Oliver Jensen

kaioliver.jensen@usz.ch

Michel Paul Johan Teuben

michel.teuben@usz.ch

Carsten Mand

mand@orthopaedie-gladenbach.de

Laura Moosdorf

lmoosdor@med.uni-marburg.de
Kai Sprengel

kai.sprengel@usz.ch

Alba Shehu

ashehu@ukaachen.de

Roman Pfeifer

roman.pfeifer@usz.ch 


\section{Introduction}

Simultaneous admission of trauma patients exposes institutions to an increased number and complexity of injuries and can lead to the overwhelming of institutions' capacities. A situation is defined as a mass casualty incident (MCI) if the number of patients and complexity of cases exceeds the ability of an institution to provide adequate care. Preparedness for an MCI is essential to optimize outcome [1-4]. The global increase of mass events, mass shootings and terrorist attacks within the last decades has further increased the frequency of MCIs in civilian settings [5-10].

Treatment guidelines during MCIs are in line with advanced trauma life support ${ }^{\circledR}$ (ATLS) and damage control principles [11, 12]. However, the simultaneous admission of seriously-injured patients are not only characterized by increased medical demands, but also by logistical difficulties. Under normal conditions, the aim of healthcare providers is to provide optimal quality of care for admitted patients. In the case of multiple trauma cases, however, resources may be lacking to do so. So, during MCIs, the utilization of resources should be dosed, and only highly urgent diagnostics and interventions should be performed. This concept is known as minimal acceptable care [10, 13].

In the USA, up to $9 \%$ of trauma patients have been reported under MCI-scenarios. This does not appear to be so frequent in Europe, potentially due to a lower number of firearm incidents $[13,14]$. As a readout for quality of care during MCIs in Europe, parallel treatment scenarios including seriously injured patients can be monitored. The outcome of patients admitted simultaneously with other trauma victims has not been studied in a multicenter setting before. Furthermore, the impact of multiple trauma cases on patient is unclear. We hypothesized that:

Steffen Ruchholtz

ruchholt@med.uni-marburg.de

Rolf Lefering

rolf.lefering@uni-wh.de

Hans-Christoph Pape

hans-christoph.pape@usz.ch

1 Department of Trauma, University Hospital Zurich, Raemistrasse 100, 8090 Zurich, Switzerland

2 Department of Surgery, Thurgau Cantonal Hospital, Frauenfeld, Switzerland

3 Department of Trauma-,Hand-, and Reconstructive Surgery, University Clinic Giessen and Marburg, Marburg, Germany

4 Institute for Research in Operative Medicine (IFOM), University Witten/Herdecke, Cologne, Germany
1. in multiple trauma admission scenarios (defined as $>1$ seriously injured individual), patients are admitted more frequently to level-one trauma centers than under regular conditions.

2. The outcome in serious injured trauma victims admitted simultaneously with other trauma patients to a single institution is impaired compared with individual trauma admissions.

\section{Materials and methods}

For this study, the TraumaRegister DGU® of the German Trauma Society (Deutsche Gesellschaft für Unfallchirurgie, DGU) was utilized. The aim of this multi-centre database is a pseudonymized and standardized documentation of severely injured patients.

Data are collected prospectively in four consecutive time phases from the site of the accident until discharge from hospital: (A) Pre-hospital phase, (B) Emergency room and initial surgery, (C) Intensive care unit and (D) Discharge. The documentation includes detailed information on demographics, injury pattern, comorbidities, pre- and in-hospital management, course on intensive care unit and outcome of each individual.

The infrastructure for documentation, data management and data analysis is provided by AUC-Academy for Trauma Surgery (AUC-Akademie der Unfallchirurgie $\mathrm{GmbH}$ ), a company affiliated to the German Trauma Society. The scientific leadership is provided by the Committee on Emergency Medicine, Intensive Care and Trauma Management (Sektion NIS) of the German Trauma Society. The participating hospitals submit their data pseudonymized into a central database via a web-based application. Scientific data analysis is approved according to a peer review procedure laid down in the publication guideline of TraumaRegister DGUß.

The present study is in line with the publication guidelines of the TraumaRegister DGU® and registered as TRDGU project ID 2018-024.

All trauma patients admitted to a German hospital between 2002 and 2015 with an ISS $\geq 9$ and treated with ATLS $®$ - algorithms were selected [15]. An ISS $\geq 9$ represents serious injury, and this threshold was chosen based on recommendations from Palmer for trauma outcome studies focussing on more factors of interest than mortality only [16]. We excluded all transfer-in-patients (i.e., only primary admissions), early transfer-out patients $(<48 \mathrm{~h}$; outcome unknown), and those individuals in which the exact time of hospital admission was unavailable. In addition, patients admitted simultaneously with more than 2 trauma patients were also excluded. 
Injuries were classified according to the Abbreviated Injury Scale (AIS, version 2005, update 2008) and Injury Severity Scores (ISS) $[15,17,18]$. Neurological status was described by the Glasgow Come Scale [19]. Utilization of two established treatment concepts has been compared as well: early total care (ETC) and DCS (damage control surgery). Application of damage control surgery was defined as the need to execute damage control surgery for at least one of the encountered injuries. Indications for damage control surgery are described in the updated German polytrauma S3-guidelines. [20]. In accordance with current recommendations for analysis of large datasets on trauma and to optimize power of the study, outcome-adjusted comparisons using the prognostic RISC II score were performed [21].

Scenarios were divided based on the presence or absence of further admission of multiple trauma patients. The following study groups were composed:

Group IND: individual/regular trauma admissions.

Group MULT: patients admitted within 60 min after another trauma admission.

Sub-group SIM: patients admitted within $10 \mathrm{~min}$ after another trauma admission.

\section{Statistics}

Continuous measurements were reported as mean with standard deviation (SD); and in case of skewed distribution of values, the median was reported in addition. ISS was documented as ISS (IQR). Categorical variables were reported as percentages with overall sample size. Due to the large group sizes, even minor differences would turn out to be statistically significant. Comparing p-values only is therefore not indicated [22]. Formal statistical testing was restricted to a few situations (chi-squared test for frequencies). Mortality rates were given with $95 \%$ confidence intervals (CI) in order to evaluate the difference to the prognosis (mean value derived from RISC II prognostic model). The RISC II is a prognostic score derived and validated with data from the TR-DGU [23]. Data were analyzed using SPSS Statistics Software (version 24, IBM Inc., Armonk, NY, USA).

\section{Results}

A total of 200,804 patients were admitted to participating hospitals between 2002 and 2015. All patients with an ISS $\geq 9$ treated in a German hospital were identified and included in the study if the date and time of admission were available $(n=147,965)$. Transferred patients $(n=15,402)$ and potential double entries $(\mathrm{n}=181)$ were excluded, leaving 132,382 patients for analysis.

During the treatment of 2,200 seriously injured trauma patients (1.7\% of 132,382), a second seriously injured trauma case has been admitted within one hour of admission. Another 62 additional patients were admitted within one hour. The total number of patients treated under 'multiple patients' conditions (MULT) was therefore 4,462 patients, representing $3.4 \%$ of all admissions. The subgroup of simultaneously admitted patients (admitted at the same time, or not more than $10 \mathrm{~min}$ later/SIM-group) consisted of 1686 cases (1.3\%).

The mean age of the patients in the multiple admissions groups was $48.0 \pm 22.3$ years and $67.7 \%$ of patients were male. No relevant differences in patient-specific characteristics between groups were observed (Table 1).

The mean / median time difference between subsequent admissions was $22 / 18 \mathrm{~min}$ in the MULT-group. The MULT patients were more frequently involved in traffic accidents than regular admitted patients (68.6\% vs. $55.8 \%)$. The individuals in the SIM-group were even more frequently involved in traffic accidents $(73.8 \%)$.

The median (IQR) ISS of the whole cohort was 17 (13-26) points. No relevant differences in injury severity were seen between study groups (Table 1). Relevant head injuries were diagnosed less frequently in MULT patients ( $37 \%$ vs. $41 \%, p<0.001)$, whereas thoracic injuries were seen more frequently ( $49 \%$ vs. $45 \%, \mathrm{p}<0.001)$. In subjects admitted simultaneously (group-SIM), relevant craniocerebral/cervical spine injuries were diagnosed in only $33.5 \%$ of cases and thoracic injuries occurred in $52.8 \%$ of the patients. Parallel to the lower rates of head injury, unconsciousness $(\mathrm{GCS} \leq 8)$ was also less frequently observed in MULT cases (18\%) and SIMultaneous (15\%) admissions.

\section{Pre-hospital parameters and interventions}

Individually admitted trauma patients were more frequently intubated out-of-hospital than MULT or SIM patients (33\% vs. $29 \%$ vs. $25 \%$ ). Pre-hospital sedation, chest-tube application and cardio-pulmonary resuscitation rates, however, did not differ between the groups.

Average transfer times from scene to hospital did not differ largely between the different conditions and varied between 63 and $65 \mathrm{~min}$. Pre-hospital data are summarized in Table 2.

\section{Level of care}

Relative admission rates to level-one trauma centers were higher in multiple seriously injured trauma victims (68.2\%), compared to regular conditions (57.9\%, 
Table 1 Patient and trauma characteristics

\begin{tabular}{|c|c|c|c|}
\hline & Individual admissions (IND) & Multiple admissions (MULT) & Simultaneous admissions (SIM) \\
\hline No. of patients & 127,920 & $4,462(3.4 \%)$ & $1,686(1.3 \%)$ \\
\hline Age (years) & $49.2(22.1)$ & $48.0(22.3)$ & $47.3(22.4)$ \\
\hline Sex ( $\%$ males) & $71.1 \%$ & $67.7 \%$ & $65.5 \%$ \\
\hline \multicolumn{4}{|l|}{ Injury type } \\
\hline Traffic accident $(\%)$ & $55.8 \%$ & $68.6 \%$ & $73.8 \%$ \\
\hline Blunt trauma $(\%)$ & $95.6 \%$ & $95.7 \%$ & $94.8 \%$ \\
\hline $\mathrm{GCS} \leq 8$ & $21.1 \%$ & $17.6 \%$ & $14.9 \%$ \\
\hline Total ISS & $17(13-26)$ & $18(13-26)$ & $17(13-26)$ \\
\hline \multicolumn{4}{|c|}{ Relevant injuries (AIS $\geq 3$ ) } \\
\hline Head & $41.1 \%$ & $37.0 \%$ & $33.5 \%$ \\
\hline Thorax & $45.0 \%$ & $49.0 \%$ & $52.8 \%$ \\
\hline Abdomen & $12.3 \%$ & $14.4 \%$ & $15.4 \%$ \\
\hline Extremities & $30.4 \%$ & $29.3 \%$ & $30.1 \%$ \\
\hline
\end{tabular}

All data presented as mean (SD) or percentages. ISS is documented as median (IQR). Simultaneous admissions are a subgroup of the multiple admission group

$p<0.001)$. Additionally, under these circumstances, patients were less frequently admitted to level-two trauma centers $(32.4 \%$ under regular scenarios and $25.6 \%$ of patients under MULTiple conditions). However, this phenomenon was not observed in the case of SIMultaneously admitted patients. Admission to level-three trauma centers is not altered during any specific scenario (Table 3).

\section{Resuscitation room characteristics, diagnostics and procedures}

Admission hemodynamics and metabolic status did not differ between groups. CT-scanning was performed in nearly $90 \%$ of cases and in all conditions. However, the time from admission to imaging was increased by $2-4 \mathrm{~min}$ in the case of MULTiple or SIMultaneous admissions, compared with individual admissions.

When comparing the duration of other imaging, a similar trend was observed for chest x-ray and sonography of the abdomen. Chest X-ray imaging seemed to be applied less frequent (40\% vs. $44 \%$, Table 3 ) in the case of MULTiple admissions. The average time in the resuscitation room was slightly increased in the case of MULTiple and SIMultaneous admissions (65.9 and $68.2 \mathrm{~min}$ vs. $64.7 \mathrm{~min}$ in regular admissions). The utilization of damage control strategies and discharge pattern from the ED was similar in all three subgroups considered.

\section{Outcome}

No relevant differences in transfusion requirements were observed between the groups. Regarding mortality the best outcome is seen in patients treated SIMultaneously. However, after adjustment for relevant risk factors summarized according to RISC II score, observed outcome was nearly identical with the respective prognosis (Table 4). In MULTiple admitted patients, the outcome was favorable for both the first patient $(n=2107$; observed $12.0 \%$; expected $11.8 \%$ ) and for second patient who was admitted during the ER treatment of the first $(n=2095$; observed: $10.8 \%$; expected: $11.3 \%$ ).

\section{Discussion}

The current study demonstrates that.

1. Simultaneous treatment of seriously injured patients in German institutions is rare; it occurs in only $3.4 \%$ of trauma admissions within the TR-DGU.

2. Patients admitted under MULTiple admission scenarios have more often been involved in traffic accidents, have craniocerebral injuries and an impaired neurological status less often, and are more frequently transferred to a level-one trauma center than with regular admissions.

3. Diagnostics in multiple admission scenario situations is slightly altered and takes longer. Nevertheless, management and outcome in patients admitted in parallel to other trauma victims is similar to individual trauma admissions.

In the USA, $9 \%$ of trauma cases are considered as MCIsituations, an even more profound category of simultaneous trauma admission conditions [14]. In the current study, 
Table 2 Prehospital care

\begin{tabular}{llll}
\hline & Individual admissions & Multiple admissions & Simultaneous admissions \\
\hline Intubation & $32.5 \%$ & $28.5 \%$ & $24.5 \%$ \\
Sedation & $71.0 \%$ & $68.5 \%$ & $69.5 \%$ \\
Chest-tube & $4.3 \%$ & $3.4 \%$ & $3.1 \%$ \\
Cardio-pulmonary resuscitation (CPR) & $3.3 \%$ & $3.3 \%$ & $2.5 \%$ \\
Volume therapy & $87.0 \%$ & $87.2 \%$ & $86.4 \%$ \\
Total volume given (mL) & $996 / 1000(697)$ & $976 / 1000(681)$ & $927 / 750(635)$ \\
Total time from accident to hospital (min) & $63 / 59(29)$ & $65 / 60(29)$ & $64 / 60(28)$ \\
\hline
\end{tabular}

All data in mean/median (SD) or percentages. Subgroup with simultaneous admissions includes consecutive trauma admissions within 10 min to the same facility

Table 3 Allocation, patient characteristics and resuscitation room diagnostics

\begin{tabular}{|c|c|c|c|}
\hline & Individual admissions & Multiple admissions & Simultaneous admissions \\
\hline \multicolumn{4}{|l|}{ Level of care (Trauma centre) } \\
\hline Level one / supra-regional & $57.9 \%$ & $68.2 \%$ & $58.4 \%$ \\
\hline Level two / regional & $32.4 \%$ & $25.6 \%$ & $32.1 \%$ \\
\hline Level three / local & $9.6 \%$ & $9.5 \%$ & $9.5 \%$ \\
\hline \multicolumn{4}{|l|}{ Admission parameters } \\
\hline Systolic blood pressure $(\mathrm{mmHg})$ & $128(31)$ & $128(31)$ & $130(31)$ \\
\hline Heart rate $(\mathrm{BPM}) *$ & $89(22)$ & $89(22)$ & $90(21)$ \\
\hline Catecholamines * & $20.3 \%$ & $17.9 \%$ & $16.7 \%$ \\
\hline Resuscitation volume $(\mathrm{mL}) *$ & $1487(1750)$ & $1326(1560)$ & $1366(1642)$ \\
\hline Serum Hemoglobin level (g/dL) & $12.8(2.5)$ & $12.8(2.5)$ & $12.9(2.4)$ \\
\hline Temperature $\left({ }^{\circ}\right.$ Celsius $) *$ & $36.1(1.2)$ & $36.3(1.0)$ & $36.3(1.1)$ \\
\hline Base Excess & $-2.2(4.7)$ & $-1.9(4.6)$ & $-1.7(4.1)$ \\
\hline Platelet counts $\times 10^{9} / \mathrm{L} *$ & $216(81)$ & $217(76)$ & $218(78)$ \\
\hline Quick's value (\%) & $85(22)$ & $85(22)$ & $87(21)$ \\
\hline Partial prothrombin time (seconds) $*$ & $32(17)$ & $31(17)$ & $31(19)$ \\
\hline \multicolumn{4}{|l|}{ Imaging } \\
\hline CT-scanning & $88.2 \%$ & $89.3 \%$ & $88.2 \%$ \\
\hline Sonography of the abdomen & $83.6 \%$ & $82.4 \%$ & $83.8 \%$ \\
\hline Chest x-ray & $44.0 \%$ & $39.7 \%$ & $40.6 \%$ \\
\hline \multicolumn{4}{|l|}{ Times to Imaging } \\
\hline Chest X-ray (min) & $14.5 / 8$ & $15.4 / 9$ & $16.8 / 10$ \\
\hline Sonography (min) & $5.9 / 4$ & $6.4 / 5$ & $7.4 / 5$ \\
\hline First $C T$ (min) & $24.0 / 20$ & $25.9 / 20$ & $28.3 / 22$ \\
\hline Total time in the ER* (min) & $64.7 / 20$ & $65.9 / 52$ & $68.2 / 55$ \\
\hline \multicolumn{4}{|l|}{ Applied initial surgical concept } \\
\hline Damage control orthopedics $*$ & $8.6 \%$ & $9.0 \%$ & $8.1 \%$ \\
\hline
\end{tabular}

All time data with mean / median (standard deviation); $C I=$ confidence interval, BPM = beats per minute

"available only for patients documented with the standard dataset

about $3.4 \%$ of trauma patients were admitted under multiple admission conditions. This difference can be explained by (1) variation in distribution of trauma facilities in metropolitan areas between both regions [10, 24, 25], (2) differences in definitions of MCIs [24, 25] or, (3) striking differences in occurrence of fire-arm 
incidents $(<5 \%$ of cases in the current study are due to penetrating trauma, whereas over 60 percent of MCI-patients in the US-study from Shoher et al. were suffering from penetrating trauma [14]).

Despite comparable injury severity, injury patterns differ slightly between groups and individually admitted trauma patients were more often diagnosed with craniocerebral injuries. This also explains the higher pre-hospital intubation rates and larger number of patients with an altered GCS score. Conversely, patients admitted simultaneous with other trauma victims had more thoracic injuries diagnosed. As anticipated, pre-hospital care was, except for different intubation rates, comparable between all groups as trauma patients are generally being treated by separate trauma teams.

Our data further show that patient allocation is altered in the case of parallel trauma cases. Under MULTiple admission scenarios, patients are more likely to be admitted to a level-one trauma center than under normal conditions. This is at the expense of level-two trauma centers which receive comparatively less patients in these cases. As level-one trauma centers are required to be able to manage the parallel treatment of multiple severely injured patients, it may be practical in some situations (i.e., car accidents) to cluster patients accordingly and admit them to the same institute. Interestingly, this discrepancy was not observed in those situations in which patients were admitted simultaneously (within $10 \mathrm{~min}$ ). Level-three trauma center admission rates are less affected, as their role in the treatment of severely injured patients in our region is minimal.

In scenarios of multiple severely injured trauma victims, adequate patient distribution is vital. Adequate distribution entails patient allocation based on optimal matching of individual patient demands with available institutional capacities in a specific region. Trauma systems are the key factors in ensuring adequate patient distribution. The German national trauma network aims to ensure trauma coverage for the entire geographic area of Germany and to achieve optimal regional and national allocation of trauma facilities. By defining strict triage criteria for hospitals with different trauma care levels, patient distribution can be optimized and mismatches in trauma care supply and demand can be minimized [26]. This is essential, both in singular and multiple trauma situations. In both scenarios, well-designed institutional protocols are mandated and, in our view, these protocols should be practiced on a regular basis through simulation training of involved personnel [27]. This recommendation is based on studies showing that programs regularly utilizing inter-professional simulation of pre- and in-hospital trauma care are associated with better overall outcomes in severe trauma.

Table 4 Outcome

\begin{tabular}{|c|c|c|c|}
\hline & Individual admissions & Multiple admissions & Simultaneous admissions \\
\hline \multicolumn{4}{|l|}{ Destination after $E R^{*}$} \\
\hline Emergency intervention & $4.2 \%$ & $4.8 \%$ & $4.7 \%$ \\
\hline Operation room & $36.8 \%$ & $35.2 \%$ & $36.7 \%$ \\
\hline Intensive care & $50.9 \%$ & $51.3 \%$ & $49.2 \%$ \\
\hline Ward & $8.0 \%$ & $8.7 \%$ & $9.4 \%$ \\
\hline \multicolumn{4}{|l|}{$\begin{array}{l}\text { Blood transfusion } \\
\text { (units of pRBC until ICU) }\end{array}$} \\
\hline None & $87.4 \%$ & $87.8 \%$ & $87.9 \%$ \\
\hline $1-9$ & $10.1 \%$ & $9.6 \%$ & $9.6 \%$ \\
\hline$\geq 10$ & $2.5 \%$ & $2.5 \%$ & $2.5 \%$ \\
\hline Mortality in hospital & $12.1 \%$ & $10.8 \%$ & $9.6 \%$ \\
\hline 24-h mortality & $6.2 \%$ & $5.6 \%$ & $5.1 \%$ \\
\hline \multicolumn{4}{|c|}{ Observed / expected mortality** } \\
\hline No. of cases & 117,754 & 4,202 & 1,526 \\
\hline Observed mortality & $13.1 \%$ & $11.4 \%$ & $10.6 \%$ \\
\hline $95 \%$ CI for mortality & {$[12.9-13.3]$} & {$[10.4-12.4]$} & {$[9.1-12.2]$} \\
\hline RISC II prognosis & $12.8 \%$ & $11.5 \%$ & $10.2 \%$ \\
\hline
\end{tabular}

All time data with mean / median (standard deviation); $C I=$ confidence interval, $B P M=$ beats per minute

*available only for patients documented with the standard dataset

** primary admitted cases with RISC II only; no early transfer out 
Furthermore, in order to improve quality of care, it is essential to analyze cases of multiple trauma admissions retrospectively by debriefing sessions and/or scientific reporting [27-29].

Regarding imaging, we demonstrated that X-ray imaging was performed less frequently on patients from the MULTiple admission group when compared with regular admissions. This can be explained by the larger percentage of patients with thoracic trauma and the subsequent need for CT-scanning. CT-scanning habits in our investigation did not differ between groups, which is in contrast to a study from Aylwin et al. in which they described minimal utilization of computed tomography imaging during the casualty-receiving phase of a MCI. They further observed a trend toward more liberal use of ultrasound investigations in trauma victims during MCI-conditions [24], whereas our study did not demonstrate altered utilization of ultrasound diagnostics.

Processing times in our study, however, were affected by the admission of multiple trauma patients. Times until CT-scanning was performed, increased in the case of multiple trauma admissions (and increased even more prominently in SIMultaneously admitted patients). Prolonged waiting times for imaging, most likely also affected the total time that patients spend on the emergency department. The encountered increased processing times are therefore most likely due to the limited availability of CT-scanning devices and the need to transfer patients from the table to the bed, as well as cleaning and preparing the CT-scanner in between investigations.

Several logistical improvements have recently been shown to improve the efficiency of the CT-scanning procedure in trauma. First, Huber-Wagner et al. showed that a short distance between the CT scanner and the emergency department (defined as less than $50 \mathrm{~m}$ ) is associated with improved outcome in severely injured patients [30]. Similar findings by Saltzherr et al. showed that a CT scanner located in the emergency department as opposed to the radiology department was associated with improved workflows [31]. Further, hybrid shock rooms equipped with an angio-embolization suite (e.g., active bleeding in pelvic injuries) can further improve efficiency and outcome in severe trauma $[32,33]$. However, not all trauma centers are able to fully implement these optimized features in their emergency departments. In order to overcome this discrepancy, especially since high-volume centers are associated with better polytrauma outcomes than smaller volume centers, adequate pre-hospital triage and well-designed trauma networks are essential [34, 35].

Of note, the impact of the encountered $10 \%$ time difference between regular and simultaneous trauma admissions on outcome was not determined by the current study, and therefore, the clinical relevance of this finding remains unclear and allows for speculation. Future studies and validation in other datasets are indicated.

The current study was the first to show that simultaneous admission of seriously injured trauma cases does not alter the utilization of treatment concepts. All relevant factors involved in decision making adapted from safe definitive surgery (SDS) recommendations were comparable between groups [36]. In line with SDS-principles, application and timing of definitive care for orthopedic fractures also did not differ between the study groups.

These findings, however, are in contrast with a study from Gonzalez et al. [37] in which during an MCI in France, an evident trend toward increased utilization of damage control orthopedics was observed.

Interestingly, mortality rates are comparable between groups and no relevant differences were found in RISCanalyzes. These findings are in line with a study from Shoher et al. in which trauma patients admitted simultaneously with other patients to their urban level-one trauma center had comparable outcomes with individual admissions [14]. Ball et al. further demonstrated that ICU-stay, morbidity and mortality were not impaired in MCI-patients admitted to a single regional trauma center. Similar to our findings, they also found that processing times were increased in MCI cases compared to regular trauma admissions [13].

Our study has some limitations. As the TraumaRegister DGU® includes large numbers of patients, their validity is limited due to the identification of statistical differences without clinical relevance. Therefore, statistic testing was only performed for specific analysis with limited datasets.

In conclusion, the current study underlines the rarity of the simultaneous admission of seriously injured trauma patients in Germany. This multicenter study further reveals specific injury patterns, the distribution of patients over different types of trauma centers in multiple trauma patient scenarios, compared with regular admissions. Moreover, simultaneous admission of multiple patients is associated with decreased efficiency of diagnostics but does not worsen outcome. Upcoming research should focus on interventions aimed to further optimize preparedness for multiple admission scenarios and thereby improving outcome under these specific conditions.

Acknowledgments Thanks to M. Collins for his assistance and language editing of the manuscript.

Funding Open Access funding provided by Universität Zürich.

\section{Compliance with ethical standards}

Conflict of interest The author declares that they have no conflict of interest. 
Open Access This article is licensed under a Creative Commons Attribution 4.0 International License, which permits use, sharing, adaptation, distribution and reproduction in any medium or format, as long as you give appropriate credit to the original author(s) and the source, provide a link to the Creative Commons licence, and indicate if changes were made. The images or other third party material in this article are included in the article's Creative Commons licence, unless indicated otherwise in a credit line to the material. If material is not included in the article's Creative Commons licence and your intended use is not permitted by statutory regulation or exceeds the permitted use, you will need to obtain permission directly from the copyright holder. To view a copy of this licence, visit http://creativecommons. org/licenses/by/4.0/.

\section{References}

1. Mistovich JJ, Karren KJ, Hafen B (2013) Prehospital Emergency Care, 10th edn. Prentice Hall, Saddle River, New Jersey

2. Ben-Ishay O, Mitaritonno M, Catena F et al (2016) Mass casualty incidents - time to engage. World J Emerg Surg 11:8

3. Amogy G, Belzberg H, Mintz Y et al (2004) Suicide bombing attacks: update and modifications to the protocol. Ann Surg 239(3):295-303

4. Rosolski T, Matthes N (2006) Notfallmanagement - organisation der medizinischen versorgung beim massenanfall von verletzten oder Erkrankten. Anesth Intensiv Notfallmed Schmerzth 41(6):370-375

5. Cushman JG, Pachter HL, Beaton HL (2003) Two New York City Hospitals" surgical response to the September 11, 2001 terrorist attack in New York city. J Trauma 54(1):147-154

6. Frykberg ER (2002) Medical management of disasters and mass casualties from terrorist bombings: how can we cope? J Trauma 53(2):201-212

7. Hogan DE, Waeckerle JF, Dire DJ et al (1999) Emergency department impact of the Oklahoma city terrorist bombing. Ann Emerg Med 34:160-167

8. Biancolini CA, Del Bosco CG, Jorge MA (1999) Argentine Jewish community institution bomb explosion. J Trauma 47:728-732

9. Edwards DS, McMenemy L, Stapley SA et al (2016) 40-years of terrorist bombings. A meta-analysis of the casualty and injury profile. Injury 47(3):642-52

10. Hirshberg A, Holcomb JB, Mattox KL (2001) Hospital trauma care in multiple-casualty incidents: a critical review. Ann Emerg Med 37:647-652

11. Taeger G, Ruchholtz S, Waydhas C et al (2005) Damage control orthopedics in patients with multiple injuries is effective, time saving, and safe. J Trauma 59(2):409-416

12. Lampi M, Vikström T, Jonson CO (2013) Triage performance of Swedish physicians using the ATLS algorithm in a simulated mass casualty incident: a prospective cross-sectional survey. Scand J Trauma Resusc Emerg Med. https://doi.org/10.1186/ 1757-7241-21-90

13. Ball CG, Kirkpatrick AW, Mulloy RH et al (2006) The impact of multiple casualty incidents on clinical outcomes. J Trauma 61(5):1036-1039

14. Shoher A, Chang DC, Efron D et al (2006) Multiple, simultaneous trauma patients: are they worse off? J Trauma 61(3):611-615

15. Baker SP, Neill BO, Haddon WJr et al (1974) Injury severity score a method for describing patients with multiple injuries and evaluating emergency care. J Trauma 14(3):187-196
16. Palmer C (2007) Major trauma and the injury severity score where should we set the bar? Annu Proc Assoc Adv Automot Med 51:13-29

17. Copes WS, Champion HR, Sacco WJ et al (1988) The injury severity score revised. J Trauma 28(1):69-77

18. Association for the Advancement of Automotive Medicine (AAAM) (2001) The Abbreviated Injury Scale 1990 Revision Update 1998. Barrington, IL

19. Teasdale G, Jennett B (1974) Assessment of coma and impaired consciousness. A Practical Scale Lancet 2:81-84

20. Bouillon B, Marzi I (2018) The updated German 'Polytraumaguideline': an extensive literature evaluation and treatment recommendations for the care of the critically injured patient. Eur J Trauma 44:1

21. Lefering $R$ (2014) Strategies for comparative analyses of registry data. Injury 45(Suppl 3):S83-S88

22. Nuzzo R (2014) Scientific method: statistical errors. Nature 506(7487):150-152

23. Lefering R, Huber-Wagner S, Nienaber U et al (2014) Update of the trauma risk adjustment model of the Traumaregister DGU: the revised injury severity classification, version II. Crit Care $18: 476$

24. Aylwin CJ, Konig TC, Brennan NW et al (2006) Reduction in critical mortality in urban mass casualty incidencts: analysis of triage, surge, and resource use after the London bombings on Juli 7 2005. Lancet 368:2219-2225

25. Committee on Trauma. Mass Casualties ACS (1999) In: Resources for optimal care of the injured patient Chicago. IL, US, pp 87-92

26. Deutsche Gesellschaft für Unfallchirurgie (2012) In: Whitebook medical care of the severely injured. 2nd revised and updated edition. Berlin, Germany.

27. Dittmar MS, Wolf P, Bigalke M et al (2018) Primary mass casualty incident triage: evidence for the benefit of yearly brief re-training from a simulation study. Scand J Trauma Resusc Emerg Med. https://doi.org/10.1186/s13049-018-0501-6

28. Kim J, Lee O (2020) Effects of a simulation-based education program for nursing students responding to mass casualty incidents: A pre-post intervention study. Nurse Educ Today 85:104297

29. Turner CD, Lockey DJ, Rehn M (2016) Pre-hospital management of mass casualty civilian shootings: a systematic literature review. Crit Care. https://doi.org/10.1186/s13054-016-1543-7

30. Huber-Wagner S, Mand C, Ruchholtz S et al (2014) Effect of the localisation of the $\mathrm{CT}$ scanner during trauma resuscitation on survival - a retrospective, multicentre study. Injury 45(Suppl 3):S76-82

31. Saltzherr TP, Bakker FC, Beenen LF et al (2012) Randomized clinical trial comparing the effect of computed tomography in the trauma room versus the radiology department on injury outcomes. Br J Surg 99(Suppl 1):105-113

32. Ito K, Nagao T, Tsunoyama T et al (2020) Hybrid emergency room system improves timeliness of angioembolization for pelvic fracture. J Trauma Acute Care Surg 88(2):314-319

33. Matsushima K, Piccinini A, Schellenberg M et al (2018) Effect of door-to-angioembolization time on mortality in pelvic fracture: every hour of delay counts. J Trauma Acute Care Surg 85(5):685-692

34. Nathens AB, Jurkovich GJ, Maier RV et al (2001) Relationship between trauma center volume and outcomes. JAMA. https://doi. org/10.1001/jama.285.9.1164

35. Cole E, Lecky F, West A et al (2016) The impact of a panregional inclusive trauma system on quality of care. Ann Surg 264(1):188-194 
36. Pape HC, Pfeifer R (2015) Safe definitive orthopaedic surgery (SDS): repeated assessment for tapered application of early definitive care and damage control? Injury 46(1):1-3

37. Gonzalez JF, Thomas J, Decroocq L et al (2019) The 14 July 2016 terrorist attack in Nice: the experience of orthopaedic surgeons. Orth Traum Surg Res 105(3):505-511
Publisher's Note Springer Nature remains neutral with regard to jurisdictional claims in published maps and institutional affiliations. 